\title{
Cigarette smoking leads to reduced relaxant responses of the cutaneous microcirculation
}

\author{
Marie-Louise Edvinsson \\ Sven E Andersson \\ Cang-Bao Xu \\ Lars Edvinsson
}

Department of Emergency Medicine, Institute of Clinical Sciences in Lund, University Hospital of Lund, Lund, Sweden
Correspondence: Cang-Bao Xu Division of Experimental Vascular Research, BMC AI3,WNC, Institute of Clinical Science in Lund, Lund University, SE-22I 84 Lund, Sweden

$\mathrm{Tel}+46462220825$

Fax +46 462220616

Email Cang-Bao.Xu@med.lu.se
Background: Smoking is a major risk factor for cardiovascular disease. The present study was undertaken to examine if cigarette smoking translates into reduced relaxant responses of the peripheral microcirculation.

Methods: The cutaneous forearm blood flow was measured by laser Doppler flowmetry. The vasodilator response to the iontophorectic administration of acetylcholine (ACh), acting via an endothelial mechanism, and sodium nitroprusside (SNP), and acting via a smooth muscle mechanism were studied. The study population consisted of 17 nonsmokers and 17 current smokers (mean age $64 \pm 2$ years, 13 females and 4 males) in each matched group.

Results: There was no difference between the groups in baseline characteristics or in basal flow. Smokers showed however significantly reduced responses to both ACh (mean \pm SEM, from $973 \pm 137 \%$ in nonsmokers to $651 \pm 114 \%$ in smokers, $p<0.05$ ) and SNP (from $575 \pm 111 \%$ in nonsmokers to $355 \pm 83 \%$ in smokers, $\mathrm{p}<0.05)$. The response to the local heating $\left(44{ }^{\circ} \mathrm{C}\right)$ was reduced in smokers (from $1188 \pm 215 \%$ in nonsmokers to $714 \pm 107 \%$ in smokers, $p<0.01$ ). In addition, there was no difference between men and women within the groups.

Conclusions: The data show that cigarette smoking results in reduced peripheral microvascular responses to both endothelial and smooth muscle cell stimulation in healthy subjects, suggesting a generalized microvascular vasomotor function.

Keywords: smoking, nonsmokers, acetylcholine, vasomotor function, cutaneous microcirculation

\section{Background}

Smoking results in 4-5 million premature deaths annually worldwide; the majority of these deaths are cardiovascular (Ezzati and Lopez 2003). Furthermore, smoking aggravates the cardiovascular risk in patients with hypertension and hyperlipidemia. In addition, it modulates the effects of other factors such as high density lipoprotein (HDL)-cholesterol (Tonstad and Svendsen 2005), low density lipoprotein (LDL) (Xu et al 1994), and insulin resistance (Tonstad and Svendsen 2005). The combination of LDL with lipid soluble smoking particles reduces the release of prostacyclin from arterial endothelial cells and enhances arterial smooth muscle cell proliferation (Xu et al 1994). This illustrates one mechanism of how cigarette smoke particles may accelerate the atherosclerotic process, produce premature atherosclerosis in epicardial coronary arteries and in large arteries in the peripheral circulation (Celermajer et al 1996; Bolinder et al 1997; Zhang et al 2006). All the above factors lead to increased risk of acute cardiovascular events, including acute myocardial infarction, stroke and sudden death.

In earlier studies, we have revealed that cigarette smoke contains lipophilic substances that are toxic to bovine endothelial and smooth muscle cells (Xu et al 1991) and can directly damage the integrity of the endothelial cell layer in man and reduce vasodilator properties in vitro (Zhang et al 2006). Thus, we hypothesize that there is a difference in microvascular reactivity in smokers and nonsmokers as tested by applying the endothelium - mediated dilator acetylcholine ( $\mathrm{ACh}$ ), the smooth 
muscle cell-mediated dilator sodium nitroprusside (SNP), or heat-induced general microvascular responsiveness. The present study was designed to examine if smoking translates to an altered microvascular reactivity in healthy middle age to elderly subjects in vivo. Iontophoresis has been proven to be a sensitive method for analysis of local microcirculation in man and can be used to test functional responses to iontophoretic administration of vasoactive substances (Andersson et al 2003).

\section{Material and methods}

\section{Population}

The material consisted of one group of current smokers $(n=17)$ and control group, of matched age and matched genders, of 17 nonsmokers (Table 1). The smokers had been smoking for no less than 5 years. In the nonsmoking group the subjects had never smoked with the exception of one subject who had been smoking before but stopped over 30 years ago. The smokers refrained from smoking on the day of study. The volunteers were randomly picked from the community registry and matched in sex and age; the subjects did not receive medication, did not have a diagnosed diabetes mellitus or known hypercholesterolemia (for demographics, see Table 1).

\section{Ethics}

All subjects gave their written informed consent prior to any examination. The protocol was approved by the Research Ethics Committee at the Faculty of Medicine, University of Lund. The investigation conformed to the principles outlined in the Declaration of Helsinki.

\section{Cutaneous forearm blood flow}

The local cutaneous forearm blood flow was measured by a laser Doppler probe. The blood flow response to the endothelium-dependent vasodilator $\mathrm{ACh}$ and the endotheliumindependent dilator SNP, both administered by iontophoresis, were determined (both agonists were obtained from Sigma Aldrich Co., St. Louis, USA). The maximum vasodilatation of the local vasculature was obtained by measuring the individual response to local warming $\left(44^{\circ} \mathrm{C}\right.$ ) (Andersson et al 2003).

\section{Laser Doppler lontophoresis}

The PeriFlux System (Perimed, Järfälla, Sweden) was used to measure cutaneous blood flow on the right dorsal side of the forearm. With this system laser-generated light is guided to the skin by a fiber optic probe. The Doppler effect is then used by the system in order to estimate blood flow. The laser
Table I The characteristics of the subjects

\begin{tabular}{llll}
\hline & Smokers & Nonsmokers & p-value \\
\hline Sex ratio female/male & $13 / 4$ & $13 / 4$ & $\mathrm{~ns}$ \\
Age $($ year $)$ & $64 \pm 2$ & $64 \pm 2$ & $\mathrm{~ns}$ \\
BMI $\left.(\mathrm{kg} \mathrm{m})^{2}\right)$ & $26 \pm \mathrm{I}$ & $26 \pm \mathrm{I}$ & $\mathrm{ns}$ \\
BP systolic $(\mathrm{mmHg})$ & $135 \pm 5$ & $125 \pm 6$ & $\mathrm{~ns}$ \\
BP diastolic $(\mathrm{mmHg})$ & $77 \pm 3$ & $68 \pm 3$ & $\mathrm{~ns}$ \\
Pulse $(\mathrm{min})$ & $72 \pm 2$ & $73 \pm 3$ & $\mathrm{~ns}$ \\
Skin temperature $\left({ }^{\circ} \mathrm{C}\right)$ & $28 \pm 0.1$ & $28 \pm 0.2$ & $\mathrm{~ns}$ \\
\hline
\end{tabular}

Notes: Two-tailed, unpaired t-test with Welch's correction was used. Data are given as mean values \pm SEM or ratio. $n=17$ in each groups, $n s=$ nonsignificant.

Doppler technique does not determine cutaneous blood flow in the ordinary sense of a volume flow per time unit ( $\mathrm{ml} / \mathrm{min}$ ). The laser Doppler technique gives spatial average of erythrocyte velocity within the superficial cutaneous capillaries. This variable most often follows cutaneous blood flow, but in cases with highly different capillary dilatation or constriction, there may be discrepancies. Therefore, the flow equivalent should be termed "cutaneous blood flow-index" or "cutaneous blood flow equivalent"; however, we have here for simplicity used the term blood flow. The data is given as perfusion unit (PU), following the convention in the field.

The subjects were asked to refrain from smoking and from consuming any beverages that contained caffeine or alcohol on the day of examination. The patient rested in a calm room and in the supine position for 15 minutes before commencing; the ambient room temperature was stable at $22-23^{\circ} \mathrm{C}$. Heart rate and blood pressure were manually measured repeatedly during the study. In addition, local skin temperature was continuously followed using an electric thermistor connected to the laser Doppler unit. The laser Doppler probe and the drug delivery system were attached to the skin. A chamber filled with ACh (2\% dissolved in MilliQ water; Sigma) was applied to the forearm. Skin temperature was noted. Basal blood flow was registered for 2 minutes. Following this, ACh (positive ion) was administered by iontophoresis $(0.2 \mathrm{~mA}$ current at the anode for 20 seconds). The stimulation was repeated 5 times with 1 minute in between. The SNP (1\% dissolved in MilliQ water) (negative ion) was applied into a new chamber ( $0.1 \mathrm{~mA}$ at the cathode for 20 seconds). The current was switched on for 1 minute to a total of 4 periods with 1 minute in between. Control experiments with application of MilliQ water alone did not reveal any significant changes in baseline flow. Microcirculation studies were randomized among the subjects, starting with either ACh or SNP.

Finally, the heat response was measured. The laser Doppler probe was heated to $44^{\circ} \mathrm{C}$ and the increase in blood flow was registered during a period of 10 minutes, which provided 
the maximum vasodilatation in the cutaneous microvessels during the test. This rise in temperature did not turn the skin red or cause any pain, however subjects occasionally reported a weak tingling or pin-prick feeling.

\section{Statistical analysis}

Two-way ANOVA with Bonferroni post-test was used for comparing dose response curves and a two-tailed, unpaired Student's t-test with Welch's correction was used to compare Hill slopes (an indicator for shift of the dose response curves). All values are given as mean \pm SEM. Values of $p<0.05$ were interpreted as statistically significant. Calculations were performed using GraphPad Prism 4 (GraphPad Software, Inc., San Diego, CA, USA).

\section{Results}

The study groups were well matched since there were no differences in general characteristics between the nonsmokers and the smokers (Figure 1, Table 1). Heart rate and blood pressure were stable throughout the measuring period, suggestive of low sympatho-adrenergic activity. In addition, local skin temperature was continuously followed and found to remain stable during the recording period.

There were no differences in baseline flow index (PU) between the two groups $(8.5 \pm 1.2$ for $\mathrm{ACh}, 12.4 \pm 1.6$ for SNP and 9.9 \pm 1.1 for heat in smokers, and $6.7 \pm 0.6$ for $\mathrm{ACh}$, $9.5 \pm 1.6$ for SNP, and $10.3 \pm 0.9$ for heat in nonsmokers; smokers vs. nonsmokers respectively, $\mathrm{p}>0.05$ ).

As shown in Figures $2 \mathrm{~A}$ and $3 \mathrm{~A}$, the agonists were administered by iontophoresis. ACh and SNP were given in repeated administrations ( 4 and 5 times, respectively, following each other) and this resulted in step-wise dilatation. The data given in Figures $2 \mathrm{~B}$ and $3 \mathrm{~B}$ show the summation of these responses in concert with the methodology of this technology. It is obvious that the smokers showed a significantly reduced response to the endothelium-dependent dilator ACh (Figure 2AB).

SNP donates nitric oxide (NO) which in turn can by pass the endothelium and has a direct effect on the smooth muscle cells causing dilatation. SNP induced stable and repeatable relaxations of the skin microcirculation and this relaxation was significantly reduced in smokers compared to nonsmokers (Figure 3AB).

The vasodilator responses to local skin heating to $44{ }^{\circ} \mathrm{C}$, which induces a general local vasodilatation, showed significantly reduced relaxations in smokers after 10 minutes of mild heating (Figure 4). After the relaxant responses had reached the maximum, the flow slowly returned to baseline.
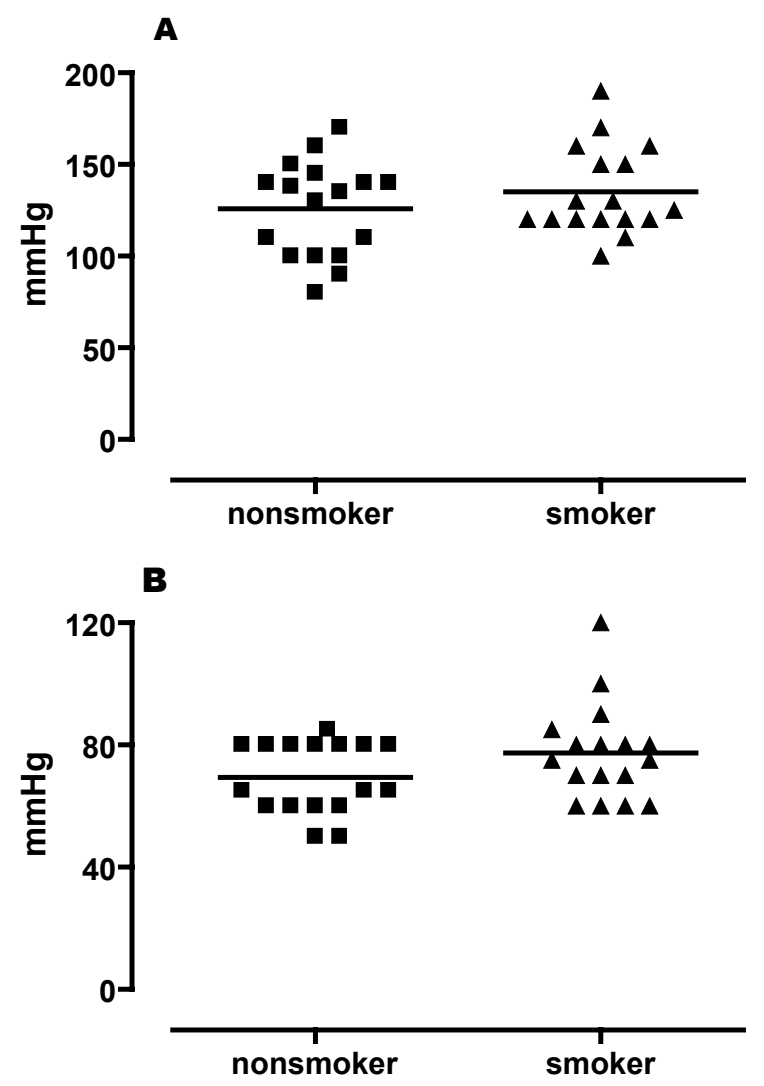

Figure I Systolic (A) and diastolic (B) blood pressure in nonsmokers and smokers. A two-tailed, unpaired Student's t-test with Welch's correction was used. There was no significant difference between the two groups neither for systolic nor for diastolic blood pressure $(n=17, p>0.05)$.

Comparison of responses of men and women within the two groups did not reveal any significant differences (data not shown). It must however be pointed out that the groups of subjects are small.

\section{Discussion}

The present study is the first to show that chronic cigarette smoking by a group of middle age to elderly subjects results in reduced relaxant responses of the cutaneous microcirculation. We observed no difference between men and women in responsiveness of the cutaneous microcirculation; however the material is small and needs to be enlarged. Tobacco smoking is one of the strongest risk factors for the development of cardiovascular morbidity and mortality (Kiyohara et al 1990; Hawkins et al 2002). It has been shown that in the United States more than $30 \%$ of all coronary heart disease deaths are attributable to cigarette smoking, with the risk being strongly dose-related (Ockene and Miller 1997). Smoking nearly doubles the risk for ischemic stroke, peripheral vascular disease, and is associated with signs of inflammation, increased intima-media thickness and formation of 
A

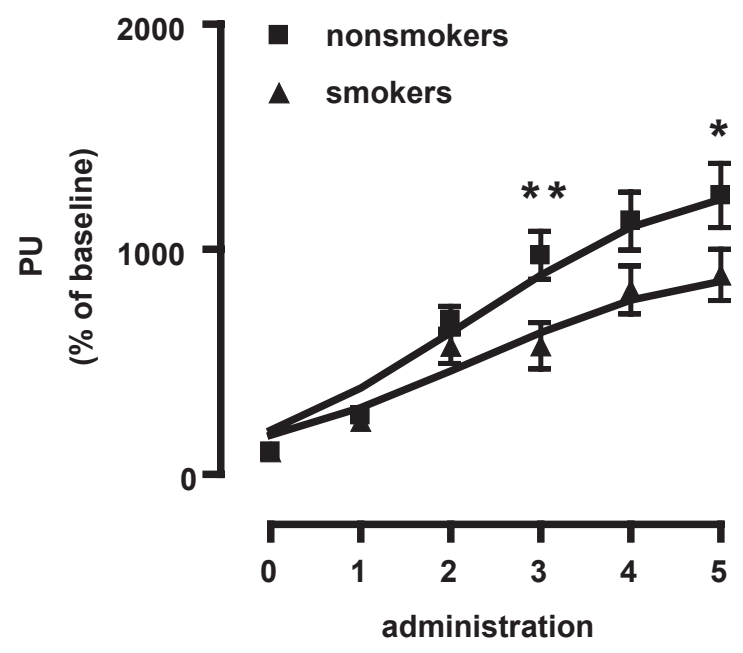

B

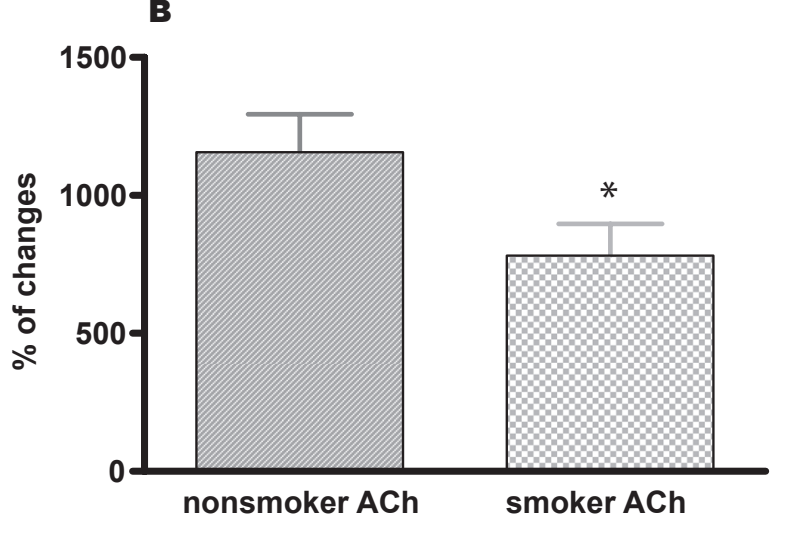

C

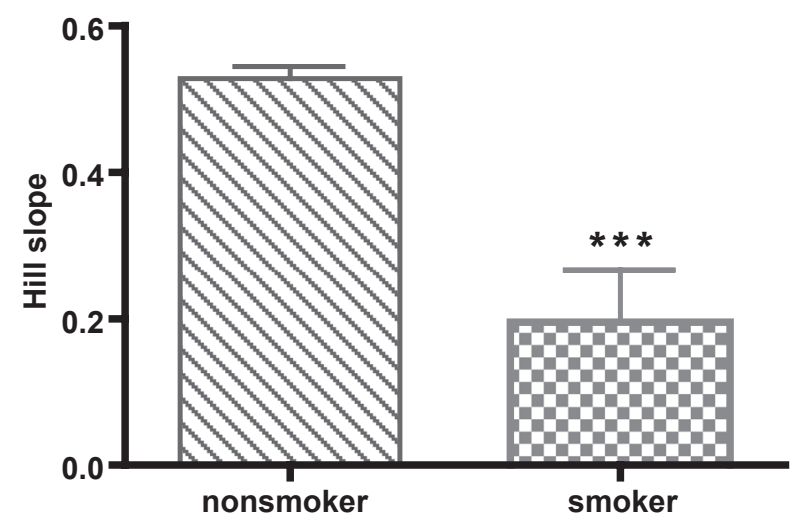

Figure 2 Skin blood flow changes (percent of perfusion units). (A) ACh-induced relaxation (given as perfusion unit) of microcirculation in nonsmokers matched with smokers, (B) percent changes of microcirculation in response to $\mathrm{ACh}$ in nonsmokers and smokers, and (C) comparing Hill slopes of the individual response curves of nonsmokers and smokers. Two-way ANOVA with Bonferroni post-test for comparing dose response curves. A two-tailed, unpaired Student's t-test with Welch's correction was used to compare Hill slopes of the response curves and percent changes of the response between non smokers and smokers. Values are given as mean \pm SEM. $\left(\mathrm{n}=17,{ }^{*} \mathrm{p}<0.05\right.$, ** $\mathrm{p}<0.01$, and $\left.{ }^{* * *} \mathrm{p}<0.001\right)$.
A

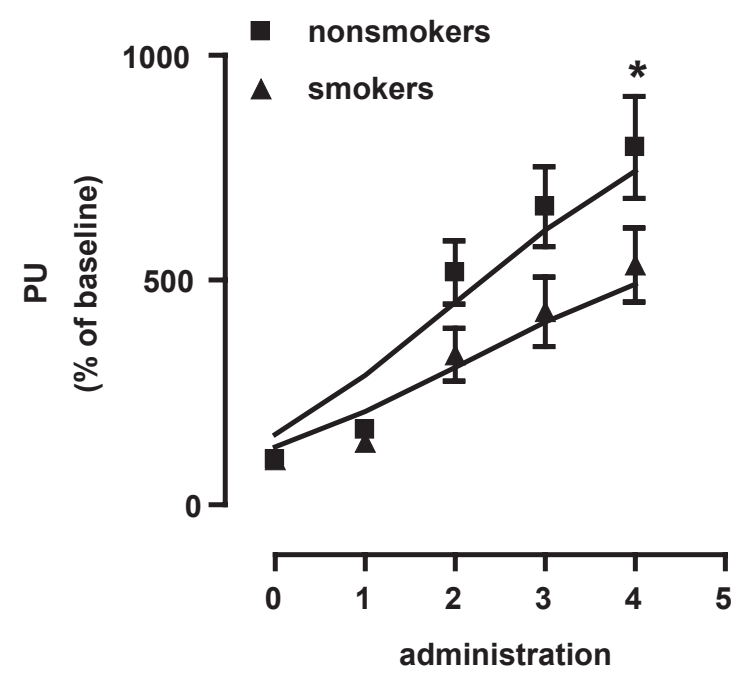

B

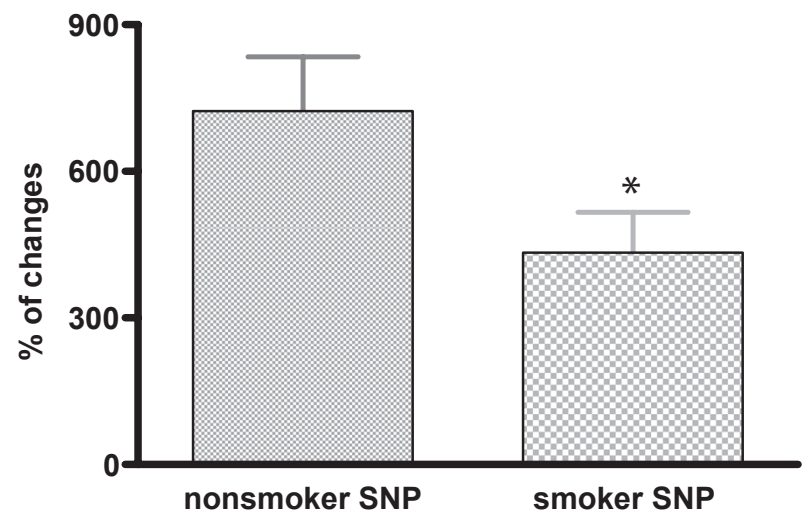

C

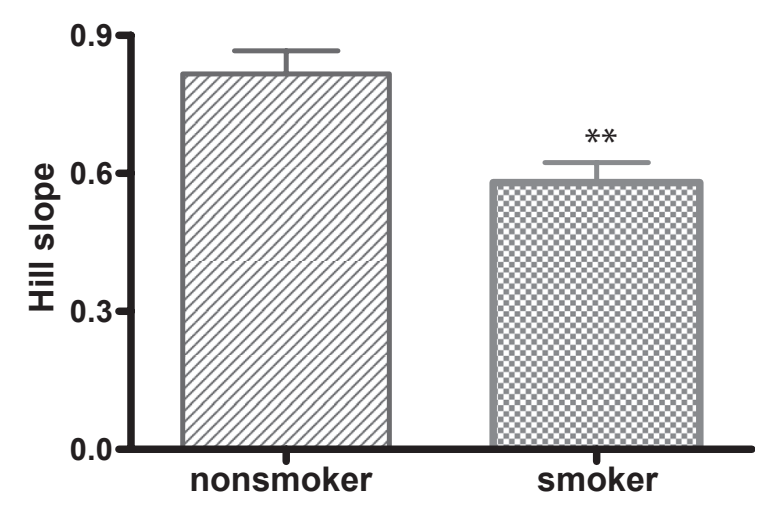

Figure 3 Skin blood flow changes (percent of perfusion units). (A) SNP-induced relaxation (given as perfusion unit) of microcirculation in nonsmokers matched with smokers, (B) percent changes of microcirculation in response to SNP in nonsmokers and smokers and (C) comparing Hill slopes of the individual response curves of nonsmokers and smokers. Two-way ANOVA with Bonferroni post-test for comparing dose response curves. A two-tailed, unpaired Student's t-test with Welch's correction was used to compare Hill slopes of the response curves and percent changes of the response between nonsmokers and smokers. Values are given as mean $\pm \mathrm{SEM}$. $\left(\mathrm{n}=17,{ }^{*} \mathrm{p}<0.05\right.$, ** $\mathrm{p}<0.01$, and $\left.* * * \mathrm{p}<0.00 \mathrm{I}\right)$. 
A
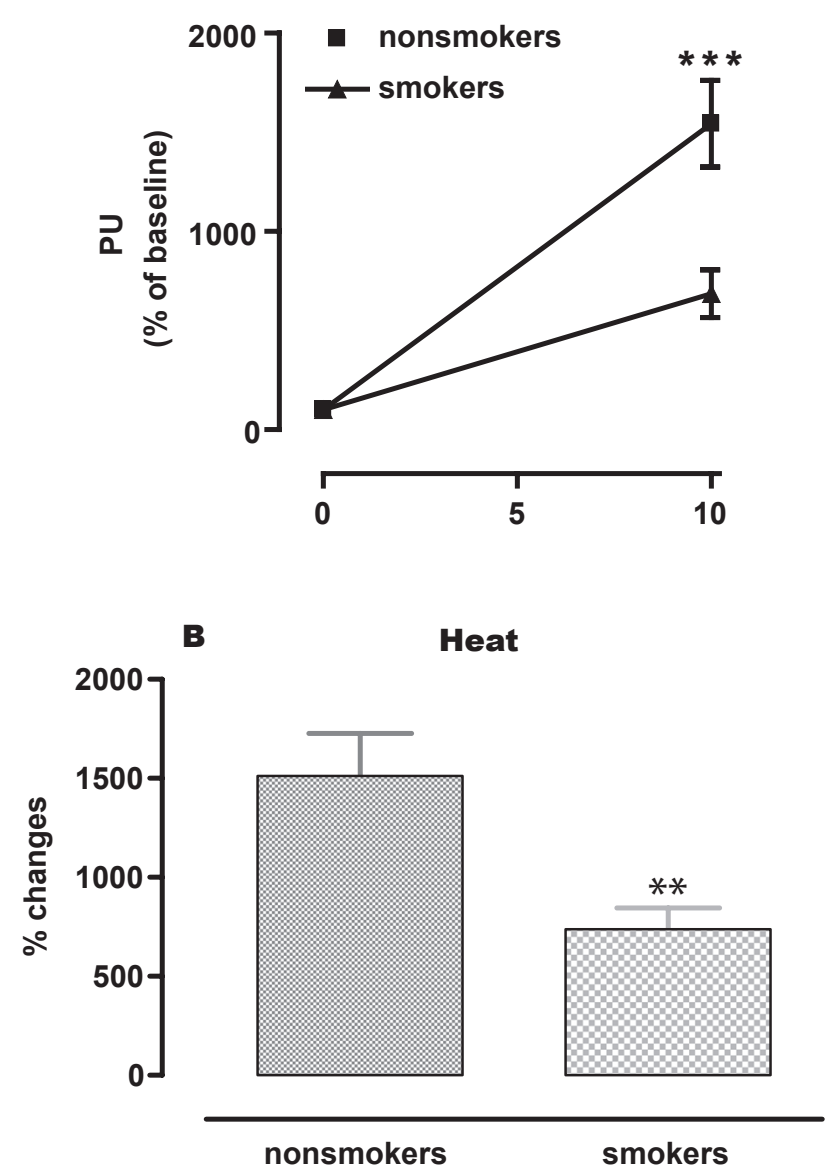

Figure 4 Skin blood flow changes (percent of perfusion units). (A) Local heating $\left(44^{\circ} \mathrm{C}\right.$ )-induced relaxation (given as perfusion unit) of microcirculation in nonsmokers matched with smokers and (B) percent changes of microcirculation in response to heating in nonsmokers and smokers. Two-way ANOVA with Bonferroni post-test for comparing response lines. A two-tailed, unpaired Student's t-test with Welch's correction was used to compare percent changes of the response between non smokers and smokers. Values are given as mean \pm s.e.m. $(n=17, p<0.00 I)$.

atherosclerotic plaques in the carotid and femoral arteries (Bolinder et al 1997).

In the present study we have focused on the functional aspects of the human peripheral microcirculation and studied the responses to ACh, SNP, and local heating in the forearm skin. Smokers showed significantly reduced responses of the local microcirculation to all three stimuli we applied. This would suggest a general change by smoking on the microvascular reactivity. The findings are in concert with the work of others. Celermajer and colleagues (1996) revealed in a group of young (age 15-30 years) healthy males that both passive and active smoking result in impaired flow mediated endothelium-dependent arterial dilatation in the brachial artery, while there was no significant alteration in nitroglycerine-induced dilatation. The impairment was considered to be due to the loss or reduction in the endothelial function since nitroglycerine (an endothelium-independent dilator) induced responses were unaltered (Celermajer et al 1996). The difference compared to our data is at least two-fold: (i) Firstly, we examined an older population (mean age $64 \pm 2$ years) with a long duration of smoking (chronic) and in predominantly women, while the previous study was carried out in young (age 15-30 years) males (Celermajer et al 1996). This may reveal the evolution of vascular changes due to smoking; first there is an attenuated endothelial function, and then a reduced general vasodilatation. Our study focused on the microcirculation while previous work has been done mainly on the larger arteries in the circulation, such as the brachial artery (Celermajer et al 1996). Those studies were invasive while we addressed the cutaneous microcirculation in a noninvasive way. It is possible that the effects of smoking occur earlier in microvessels. Rehill and collegues showed that chronic tobacco smoking is associated with an endothelial dysfunction with enhanced arterial stiffness in large arteries (Rehill et al 2006). Interestingly, these functions seemed to be partially reversible.

Our study is in agreement with the work of Pellaton and colleagues (2002) who studied the forearm local skin blood flow response to iotophoretically applied ACh and SNP in two groups of healthy male volunteers (20-35 years and 40-60 years of age). In older smokers, both ACh and SNP responses were significantly attenuated in comparison with nonsmokers. However, there was no significant difference between younger smokers and nonsmokers (Pellaton et al 2002). The differences in the technologies used, however, are notable; we applied a low current that was without effect upon the laser Doppler flow index (thus avoiding pre-treatment with local anesthetics (Pellaton et al 2002)). In addition, the iontophoresis and the laser Doppler flow index measurements were carried out simultaneously, allowing stepwise administration of agonists (not only the use of single very high dose as above). Our results are in agreement with another study in young healthy volunteers (Vecchia et al 2004). They observed that smokers were characterized by a complex alteration in the regulation of peripheral microcirculatory; inappropriate resting vasodilatation, impaired endothelium-dependent and independent vasodilatations, and paradoxical recovery of endothelium-dependent vasodilatation in response to acute smoking (Vecchia et al 2004). However, the inappropriate resting vasodilatation has not been seen by others or in our study (Table 1). In habitual young smokers, the ACh- and SNP- induced palmar skin responses were reduced, which agrees with our data. We have here confirmed these observations on an older population and in addition found that there 
was no gender difference. The detrimental effect of smoking is robust and respects neither age nor gender.

Dysfunction to endothelial and smooth muscle cells is a key step in the development of cardiovascular disease. ACh-induced endothelium-dependent relaxation consists of nitric oxide-, prostacyclin- and endothelium-derived hyperpolarization factor (EDHF)-mediated responses (Alm et al 2002). Although we and others (Pellaton et al 2002) have observed that the reductions of ACh- and SNP-induced relaxations are similar in degree, the heat-induced relaxation was larger than either of the individual responses to ACh and SNP. This suggests that in addition to an effect on the endothelial function, smoking also reduces the general smooth muscle relaxation. It is hard to determine which substances in the cigarette smoke cause the dysfunction of the arterial cells since smoking particles represent a heterogeneous group consisting of thousands of chemical substances (KnörrWittmann et al 2005). However, in vitro studies have shown that the smoking particles have many effects on endothelial and smooth muscle cells; eg, reduce prostacyclin release and eNOS expression in endothelial cells (Sun et al 1998), induce smooth muscle cell proliferation (Xu et al 1994) and enhance expression of vascular cell adhesion molecular (Chen et al 2004). These mechanisms might be involved in the reduced responsiveness of the cutaneous microcirculation by cigarette smoking in vivo and in the formation of atherosclerotic lesions.

\section{Conclusions}

The present study has revealed that smoking results in reduced microvascular relaxant responses to both specific endothelial and nonendothelial stimulations in middle age to elderly subjects. The effect of chronic smoking is stunning and strong, causing a generalized reduced relaxant responsiveness of the human peripheral microcirculation in both men and women.

\section{Disclosure}

The authors declare that they have no conflicts of interest.

\section{Acknowledgments}

This work is supported by A Clinical Innovator Award from the Flight Attendant Medical Research Institute (USA).

\section{References}

Alm R, Edvinsson L, Malmsjo M. 2002. Organ culture: a new model for vascular endothelium dysfunction. BMC Cardiovasc Disord, 2:8.

Andersson SE, Edvinsson ML, Edvinsson L. 2003. Cutaneous vascular reactivity is reduced in aging and in heart failure: association with inflammation. Clin Sci (Lond), 105:699-707.

Bolinder G, Norén A, de Faire U, et al. 1997. Smokeless tobacco use and atherosclerosis: an ultrasonographic investigation of carotid intima media thickness in healthy middle-aged men. Atherosclerosis, 132:95-103.

Celermajer DS, Adams MR, Clarkson P, et al. 1996. Passive smoking and impaired endothelium-dependent arterial dilatation in healthy young adults. $N$ Engl J Med, 334:150-4.

Chen HW, Chien ML, Chaung YH, et al. 2004. Extracts from cigarette smoke induce DNA damage and cell adhesion molecule expression through different pathways. Chem Biol Interact, 150:233-41.

Ezzati M, Lopez AD. 2003. Estimates of global mortality attributable to smoking in 2000. Lancet, 362:847-52.

Hawkins BT, Brown RC, Davis TP. 2002. Smoking and ischemic stroke: a role for nicotine? Trends Pharmacol Sci, 23:78-82.

Kiyohara Y, Ueda K, Fujishima M. 1990. Smoking and cardiovascular disease in the general population in Japan. J Hypertens Suppl, 8:S9-15.

Knörr-Wittmann C, Hengstermann A, Gebel S, et al. 2005. Characterization of Nrf2 activation and heme oxygenase-1 expression in NIH3T3 cells exposed to aqueous extracts of cigarette smoke. Free Radic Biol Med, 39:1438-48.

Ockene IS, Miller NH. 1997. Cigarette smoking, cardiovascular disease, and stroke: a statement for healthcare professionals from the American Heart Association. American Heart Association Task Force on Risk Reduction. Circulation, 96:3243-7.

Pellaton C, Kubli S, Feihl F, et al. 2002. Blunted vasodilatory responses in the cutaneous microcirculation of cigarette smokers. Am Heart $J$, 144:269-74.

Rehill N, Beck CR, Yeo KR, et al. 2006. The effect of chronic tobacco smoking on arterial stiffness. Br J Clin Pharmacol, 61:767-73.

Su Y, Han W, Giraldo C, et al. 1998. Effect of cigarette smoke extract on nitric oxide synthase in pulmonary artery endothelial cells. Am J Respir Cell Mol Biol, 19:819-25.

Tonstad S, Svendsen M. 2005. Premature coronary heart disease, cigarette smoking, and the metabolic syndrome. Am J Cardiol, 96:1681-5.

Vecchia DL, Palombo C, Ciardetti M, et al. 2004. Contrasting effects of acute and chronic cigarette smoking on skin microcirculation in young healthy subjects. J Hypertens, 22:129-35.

Xu CB, Pessah-Rasmussen H, Stavenow L. 1991. Interactions between cultured bovine arterial endothelial and smooth muscle cells: effects of injury on the release of growth stimulating and growth inhibiting substances. Pharmacol Toxicol, 69:195-200.

Xu CB, Stavenow L, Pessah-Rasmussen H. 1994. Interactions between cultured bovine arterial endothelial and smooth muscle cells; effects of modulated low density lipoproteins on cell proliferation and prostacyclin release. Scand J Clin Lab Invest, 54:191-8.

Zhang JY, Cao YX, Xu CB, et al. 2006. Lipid-soluble smoke particles damage endothelial cells and reduce endothelium-dependent dilatation in rat and man. BMC Cardiovasc Disord, 6:3. 